RETRACTION

doi:10.1038/nature13598

\title{
Retraction: Stimulus-triggered fate conversion of somatic cells into pluripotency
}

Haruko Obokata, Teruhiko Wakayama, Yoshiki Sasai, Koji Kojima, Martin P. Vacanti, Hitoshi Niwa, Masayuki Yamato \& Charles A. Vacanti

Nature 505, 641-647 (2014); doi:10.1038/nature12968

Several critical errors have been found in our Article and Letter (http:// dx.doi.org/10.1038/nature12969), which led to an in-depth investigation by the RIKEN Institute. The RIKEN investigation committee has categorized some of the errors as misconduct (see Supplementary Data 1 and Supplementary Data 2). Additional errors identified by the authors that are not discussed in RIKEN's report are listed below.

(1) Figure la and $b$ in the Letter both show embryos generated from STAP cells, not a comparison of ES- and STAP-derived chimaeric embryos, as indicated in the legend.

(2) Extended Data Fig. 7d in the Article and Extended Data Fig. 1a in the Letter are different images of the same embryo and not, as indicated in the legends, a diploid chimaera embryo and tetraploid chimaera embryo. (3) There is an erroneous description in Fig. 1a in the Letter. The right panel of Fig. 1a is not a 'long exposure' image at the camera level but a digitally enhanced one.

(4) In Fig. 4b of the Letter, STAP cell and ES cell are wrongly labelled in a reverse manner.

(5) In the Article, one group of STAP stem cells (STAP-SCs) was reported as being derived from STAP cells induced from spleens of $F_{1}$ hybrids from the cross of mouse lines carrying identical cag-gfp insertions in chromosome 18 in the background of 129/Sv and B6, respectively, and that they were maintained in the Wakayama laboratory. However, further analysis of the eight STAP-SC lines indicates that, while sharing the same $129 \times \mathrm{B}_{6} \mathrm{~F}_{1}$ genetic background, they have a different GFP insertion site. Furthermore, while the mice used for STAP cell induction are homozygous for the GFP transgene, the STAP-SCs are heterozygous. The GFP transgene insertion site matches that of the mice and ES cells kept in the Wakayama laboratory. Thus, there are inexplicable discrepancies in genetic background and transgene insertion sites between the donor mice and the reported STAP-SCs.

We apologize for the mistakes included in the Article and Letter. These multiple errors impair the credibility of the study as a whole and we are unable to say without doubt whether the STAP-SC phenomenon is real. Ongoing studies are investigating this phenomenon afresh, but given the extensive nature of the errors currently found, we consider it appropriate to retract both papers.

Supplementary Information is available in the online version of this Retraction. 